\title{
ANALYSIS OF A SIGNORINI PROBLEM WITH NONLOCAL FRICTION IN THERMO-PIEZOELECTRICITY
}

\author{
H. Benaissa, EL-H. Essoufi And R. FAKhaR \\ University Hassan 1, Morocco
}

\begin{abstract}
We consider a mathematical model which describes the frictional unilateral contact between a thermo-piezoelectric body and a rigid electrically conductive foundation. The thermo-piezoelectric constitutive law is assumed to be nonlinear and the contact is modeled with the Signorini condition, the nonlocal Coulomb friction law with slip dependent friction coefficient and the regularized electrical and thermal conductivity conditions. The variational form of this problem is a coupled system which consists of a nonlinear variational inequality for the displacement field and two nonlinear variational equations for the electric potential and the temperature. The existence of a unique weak solution to the problem is proved by using abstract results for elliptic variational inequalities and fixed point arguments.
\end{abstract}

\section{INTRODUCTION}

The piezoelectricity represents the interaction between the electrical and mechanical characteristics of some materials like crystals and ceramics. In piezoelectric materials an electrical voltage is developed when mechanical loading is applied, that's a direct piezoelectric effect. In a converse effect, the piezoelectric materials undergo mechanical strain when a voltage is applied across them. The two effects form the fundamental basis for the use of piezoelectric materials, both as sensors and strain actuators. The electroelastic characteristics of piezoelectric materials have been studied extensively and their dependence on temperature is well established. Currently, it is

2010 Mathematics Subject Classification. 35J85, 47J20, 49J40, 74F15, 74G30, 74M10, 74M15, 74S05.

Key words and phrases. Static frictional contact, thermopiezoelectric material, Signorini conditions, Coulomb's friction, frictional heat generation, variational inequality, variational analysis, fixed point process. 
interesting to incorporate the thermal effects in the electromecanical models. The resulting thermoelectromechanical models include temperature as an additional state variable to account the thermal effects in addition to the piezoelectric effects.

The literature concerning the modeling in piezoelectricity is very rich, see for example $[2,7,13]$. General models for elastic materials with thermopiezoelectric effects can be found in $[4,11,14]$ and, more recently, in [1]. Some theoretical results for static frictional contact models taking into account the interaction between the electric and the mechanic fields have been obtained in $[3,8,9,12]$, under the assumption that the foundation is insulated, and in $[5,6,10]$ under the assumption that the foundation is electrically conductive.

This work deals a new mathematical model which describes the frictional contact between a thermo-piezoelectric body and a conductive foundation. The novelty of this model consists in the thermo-electro-elastic behavior of the body and the thermal contact conditions. The motivation of this approach is that the thermal effects, such as thermal deformation and pyroelectric effects, are especially important for many smart ceramic materials. So, it may be impossible to predict the electromechanical behavior without taking into account these effects. Our interest is to describe a mathematical model in which contact, friction and thermo-piezoelectric effects are involved. To this end we will study a static problem of frictional contact, under small deformations hypothesis, wherein the material's behavior is modeled by a nonlinear thermo-electro-elastic constitutive law and the contact is described by Signorini's condition, by nonlocal Coulomb friction law of dry friction where the coefficient of friction depends on the slip and by regularized electrical and thermal conductivity conditions. To our knowledge, this model has not been studied yet and no result has been obtained for this type problem. The unique weak solvability of the associated variational formulation will be established.

The paper is structured as follows. In Section (2) we state the model of equilibrium process of the thermo-elctro-elastic body in frictional contact with a conductive rigid foundation. In Section (3) we introduce the notation, we list the assumptions on problem's data, we derive the variational formulation of the problem and also present our main result stated in Theorem(3.1). The proof of the theorem is provided in Section (4), where it is carried out in several steps and is based on arguments of variational inequalities and a Schauder fixed point theorem.

\section{Problem Statement}

We consider a piezoelectric body that occupies an open bounded subset $\Omega$ in $\mathbb{R}^{d}$ with $d=2$ or 3 . The boundary $\Gamma=\partial \Omega$ is assumed to be Lipschitz continuous, divided into three open disjoint parts $\Gamma_{1}, \Gamma_{2}$ and $\Gamma_{3}$ on the one hand and a partition of $\Gamma_{1} \cup \Gamma_{2}$ into two open parts $\Gamma_{a}$ and $\Gamma_{b}$ on the other 
hand, such that meas $\left(\Gamma_{1}\right)>0$ and meas $\left(\Gamma_{a}\right)>0$. This body is supposed to be stress free and at a free temperature. Here the temperature variations, accompanying the deformations, produce changes in the material parameters which are considered as depending on temperature. We assume that the body is fixed on $\Gamma_{1}$ where the displacement field vanishes, body forces of density $f_{0}$ act on $\Omega$, a surface traction of density $f_{2}$ acts on $\Gamma_{2}$, a volume electric charge of density $q_{0}$ acts on $\Omega$, a surface electric charge of density $q_{2}$ acts on $\Gamma_{b}$, the electrical potential vanishes on $\Gamma_{a}$ and the temperature is assumed to be zero on $\Gamma_{1} \cup \Gamma_{2}$. Moreover, the body is subjected to a volume heat source $q_{t h}$ and it comes on $\Gamma_{3}$ in friction contact with an electrically and thermally conductive obstacle, the so-called foundation.

Here and below, to simplify the notation, we do not indicate the dependence of various functions on the spatial variable $x \in \bar{\Omega}$, the indices $i, j, k, l$ take values in $\{1, \ldots, d\}$, the summation convention over repeated indices is used and the index that follows a comma indicates a partial derivative with respect to the corresponding component of the spatial variable $u_{i, j}=\partial u_{i} / \partial x_{j}$. We denote by $\mathbb{S}^{d}$ the space of second order symmetric tensors on $\mathbb{R}^{d}$ or equivalently, the space of real symmetric matrices of order $d$. We define the inner products and the corresponding norms on $\mathbb{R}^{d}$ and $\mathbb{S}^{d}$ by

$$
\begin{array}{ll}
u \cdot v=u_{i} \cdot v_{i} ; \quad\|v\|=(v \cdot v)^{\frac{1}{2}} \quad \forall u, v \in \mathbb{R}^{d}, \\
\sigma \cdot \tau=\sigma_{i j} \cdot \tau_{i j} ; \quad\|\tau\|=(\tau \cdot \tau)^{\frac{1}{2}} \quad \forall \sigma, \tau \in \mathbb{S}^{d} .
\end{array}
$$

Since the boundary $\Gamma$ is sufficiently regular, the unit outward normal field $n$ on $\Gamma$ is defined. Then the normal and the tangential components of displacement vector and stress on the boundary are

$$
\begin{aligned}
v_{n} & =v \cdot n ; \quad v_{\tau}=v-v_{n} n, \\
\sigma_{n} & =\sigma n \cdot n ; \quad \sigma_{\tau}=\sigma n-\sigma_{n} n .
\end{aligned}
$$

Under these conditions, the mechanical problem may be formulated as follows. Problem (P) : Find a displacement field $u: \Omega \rightarrow \mathbb{R}^{d}$, a stress field $\sigma: \Omega \rightarrow \mathbb{S}$, an electric potential $\varphi: \Omega \rightarrow \mathbb{R}$, an electric displacement field $D: \Omega \rightarrow \mathbb{R}^{d}$, a temperature field $\theta: \Omega \rightarrow \mathbb{R}$ and the heat flux $q: \Omega \rightarrow \mathbb{R}^{d}$ such that

$$
\begin{aligned}
& \sigma=\mathfrak{F} \varepsilon(u)-\mathcal{E}^{*} E(\varphi)-\mathcal{M} \theta \text { in } \Omega, \\
& D=\mathcal{E} \varepsilon(u)+\beta E(\varphi)+\mathcal{P} \theta \quad \text { in } \Omega, \\
& q=-\mathcal{K} \nabla \theta \quad \text { in } \Omega, \\
& \operatorname{Div} \sigma+f_{0}=0 \quad \text { in } \Omega, \\
& \operatorname{div} D=q_{0} \quad \text { in } \Omega, \\
& \operatorname{div} q=q_{t h} \quad \text { in } \Omega, \\
& u=0 \quad \text { on } \Gamma_{1}, \\
& \sigma n=f_{2} \quad \text { on } \Gamma_{2},
\end{aligned}
$$




$$
\begin{aligned}
& \sigma_{n}(u) \leq 0, \quad u_{n} \leq 0, \quad \sigma_{n}(u) u_{n}=0 \quad \text { on } \Gamma_{3}, \\
& \left\{\begin{array}{l}
\left\|\sigma_{\tau}\right\| \leq \mu\left(\left\|u_{\tau}\right\|\right)\left|R \sigma_{n}(u)\right| \\
\left\|\sigma_{\tau}\right\|<\mu\left(\left\|u_{\tau}\right\|\right)\left|R \sigma_{n}(u)\right| \Rightarrow u_{\tau}=0 \\
\sigma_{\tau}=-\mu\left(\left\|u_{\tau}\right\|\right)\left|R \sigma_{n}(u)\right| \frac{u_{\tau}}{\left\|u_{\tau}\right\|} \Rightarrow u_{\tau} \neq 0
\end{array} \quad \text { on } \Gamma_{3},\right. \\
& \varphi=0 \quad \text { on } \Gamma_{a}, \\
& D \cdot n=q_{2} \quad \text { on } \Gamma_{b}, \\
& \begin{array}{l}
D \cdot n=\psi\left(u_{n}\right) \phi_{L}\left(\varphi-\varphi_{0}\right) \quad \text { on } \Gamma_{3}, \\
\theta=0 \quad \text { on } \Gamma_{1} \cup \Gamma_{2},
\end{array} \\
& q \cdot n=k_{c}\left(u_{n}\right) \phi_{L}\left(\theta-\theta_{f}\right) \quad \text { on } \Gamma_{3} .
\end{aligned}
$$

Here, equations (2.1)-(2.2) represent the thermo-electro-elastic constitutive law of the material in which $\sigma=\left(\sigma_{i j}\right)$ denotes the stress tensor, $\varepsilon(u)$ is the linearized strain tensor, $E(\varphi)$ is the electric field, $\mathfrak{F}$ is the nonlinear elasticity operator, $\beta=\left(\beta_{i j}\right)$ is the electric permittivity tensor, $\mathcal{E}=\left(e_{i j k}\right)$ represents the third-order piezoelectric tensor, $\mathcal{E}^{*}$ is its transpose, $\mathcal{M}=\left(m_{i j}\right)$ and $\mathcal{P}=\left(p_{i}\right)$ are, respectively, the thermal expansion and the pyroelectric tensors. We recall that $\varepsilon(u)=\left(\varepsilon_{i j}(u)\right)=\left(\frac{1}{2}\left(u_{i, j}+u_{j, i}\right)\right)$ and $E(\varphi)=-\nabla \varphi=-\left(\varphi_{, i}\right)$. Notice that (2.1) takes into account the dependence of the stress on the electric potential and the temperature and (2.2) describes a linear dependence of the electric displacement on the strain, the electric potential and the temperature (see $[3,14]$ for details). The equation $(2.3)$ is the Fourier law of heat conduction where $\mathcal{K}=\left(k_{i j}\right)$ denotes the thermal conductivity tensor. The equation (2.4)-(2.6) represent the equilibrium equations for the stress, the electric displacement and the heat flux fields where $\operatorname{Div} \sigma=\left(\sigma_{i j, j}\right)$ and $\operatorname{div} D=\left(D_{i, i}\right)$ denote the divergence operator, respectively, for tensor and vector valued functions. The relations (2.7)-(2.8), (2.11)-(2.12) and (2.14) represent the mechanical, the electrical and the thermal boundary conditions. The unilateral boundary conditions (2.9) represent the Signorini law and (2.10) represent the coulomb's friction law in which $\mu$ is the coefficient of friction and $R$ is a regularization operator. The equation (2.15) represent the heat flux condition where $\theta_{F}$ is the foundation's temperature and $k_{c}$ is the coefficient of heat exchange between it and the body (see [16]). The function $k_{c}$ is assumed to depend on the normal pressure such that there is no heat flux at points where there is no contact $\left(k_{c}\left(u_{n}\right)=0\right.$ for $u_{n}<0$ and $k_{c}\left(u_{n}\right) \geq 0$ otherwise). The relation (2.13) represents the regularization of the electrical contact condition on $\Gamma_{3}$ (see [15]). Finally, the function $\phi_{L}$, used in (2.13) and (2.15), is the truncation function

$$
\phi_{L}(s)=\left\{\begin{aligned}
-L & \text { if } s<-L \\
s & \text { if }-L \leq s \leq L, \text { where } L \text { is a large positive constant. } \\
L & \text { if } s>L
\end{aligned}\right.
$$




\section{VARIATIONAL FORMULATION AND THE MAIN RESUlT}

To derive the variational formulation of our problem, we consider the real Hilbert spaces

$$
\begin{array}{lrl}
H & =\left\{u=\left(u_{i}\right), u_{i} \in L^{2}(\Omega)\right\}, & H_{1}=\{u \in H, \varepsilon(u) \in \mathcal{H}\}, \\
\mathcal{H} & =\left\{\sigma=\left(\sigma_{i j}\right), \sigma_{i j}=\sigma_{j i} \in L^{2}(\Omega)\right\}, & \mathcal{H}_{1}=\{\sigma \in \mathcal{H}, \operatorname{Div} \sigma \in H\},
\end{array}
$$

endowed with the inner products

$$
\begin{aligned}
(u, v)_{H} & =\int_{\Omega} u_{i} v_{i} d x, & (u, v)_{H_{1}}=(u, v)_{H}+(\varepsilon(u), \varepsilon(v))_{\mathcal{H}}, \\
(\sigma, \tau)_{\mathcal{H}} & =\int_{\Omega} \sigma_{i j} \tau_{i j} d x, & (\sigma, \tau)_{\mathcal{H}_{1}}=(\sigma, \tau)_{\mathcal{H}}+(\operatorname{Div} \sigma, \operatorname{Div} \tau)_{\mathcal{H}},
\end{aligned}
$$

and the associated norms $\|\cdot\|_{H},\|\cdot\|_{H_{1}},\|\cdot\|_{\mathcal{H}},\|\cdot\|_{\mathcal{H}_{1}}$. Let $H_{\Gamma}=H^{1 / 2}(\Gamma)^{d}$, $H_{\Gamma}^{\prime}$ its dual and $\langle\cdot, \cdot\rangle$ the duality pairing between the two spaces. Let also $\gamma: H_{1} \rightarrow H_{\Gamma}$ be the trace map. For every $v \in H_{1}$, we denote the trace $\gamma v$ of $v$ on $\Gamma$, again by $v$. For every $\sigma \in \mathcal{H}_{1}$, there exists an element $\sigma n \in H_{\Gamma}^{\prime}$ satisfying the following Green formula

$$
\langle\sigma n, \gamma v\rangle=(\sigma, \varepsilon(v))_{\mathcal{H}}+(\operatorname{Div} \sigma, v)_{H}, \quad \forall v \in H_{1} .
$$

Moreover, if $\sigma$ is continuously differentiable on $\bar{\Omega}$, then

$$
\langle\sigma n, \gamma v\rangle=\int_{\Gamma} \sigma n \cdot \gamma v d a, \quad \forall v \in H_{1},
$$

where $d a$ is the surface element.

Let us now consider the closed subspace of $H_{1}$ defined by

$$
V=\left\{v \in H_{1} ; v=0 \text { on } \Gamma_{1}\right\}
$$

and $K$ be the set of admissible displacements

$$
K=\left\{v \in V, v_{n} \leq 0 \text { on } \Gamma_{3}\right\}
$$

Since meas $\left(\Gamma_{1}\right)>0$, the following Korn's inequality holds

$$
\|\varepsilon(v)\|_{\mathcal{H}} \geq c_{k}\|v\|_{H_{1}}, \quad \forall v \in V .
$$

where $c_{k}>0$ is a constant which depends only on $\Omega$ and $\Gamma_{1}$. Therefore the space $V$ endowed with the inner product $(u, v)_{V}=(\varepsilon(u), \varepsilon(v))_{\mathcal{H}}$ is a real Hilbert space and its associated norm $\|v\|_{V}=\|\varepsilon(v)\|_{\mathcal{H}}$ is equivalent on $V$ to the usual norm $\|\cdot\|_{H_{1}}$. By the Sobolev's trace theorem, there exists a constant $c_{0}>0$ which depends only on $\Omega, \Gamma_{3}$ and $\Gamma_{1}$ such that

$$
\|v\|_{L^{2}\left(\Gamma_{3}\right)^{d}} \leq c_{0}\|v\|_{V}, \quad \forall v \in V .
$$


We also introduce the spaces

$$
\begin{aligned}
& Q=\left\{\eta \in H^{1}(\Omega) \mid \eta=0 \text { on } \Gamma_{1} \cup \Gamma_{2}\right\} \\
& W=\left\{\psi \in H^{1}(\Omega) \mid \psi=0 \text { on } \Gamma_{a}\right\} \\
& \mathcal{W}=\left\{D=\left(D_{i}\right) \in H_{1} \mid D_{i} \in L^{2}(\Omega), D_{i, i} \in L^{2}(\Omega)\right\} .
\end{aligned}
$$

On $Q$ and $W$ we consider the inner products and the corresponding norms given by

$$
\begin{aligned}
& \left.(\theta, \eta)_{Q}=(\theta, \eta)_{H^{1}(\Omega)}, \quad \quad\|\eta\|_{Q}=\| \eta\right) \|_{H^{1}(\Omega)}, \quad \text { for all } \theta, \eta \in Q \text {, } \\
& \left.(\varphi, \psi)_{W}=(\varphi, \psi)_{H^{1}(\Omega)}, \quad\|\psi\|_{W}=\| \psi\right) \|_{H^{1}(\Omega)}, \quad \text { for all } \varphi, \psi \in W .
\end{aligned}
$$

Since meas $\left(\Gamma_{1} \cup \Gamma_{2}\right)$ and meas $\left(\Gamma_{a}\right)$ are positive, it follows that $\left(Q,\|\cdot\|_{Q}\right)$ and $\left(W,\|\cdot\|_{W}\right)$ are Hilbert spaces.

The spaces $\mathcal{W}$ is real Hilbert space with the inner product

$$
(D, E)_{\mathcal{W}}=(D, E)_{L^{2}(\Omega)^{d}}+(\operatorname{div} D, \operatorname{div} E)_{L^{2}(\Omega)},
$$

The associated norm will be denoted by $\|\cdot\| \mathcal{W}$.

Moreover, by the Sobolev trace theorem, there exist two positive constants $c_{1}$ and $c_{2}$ such that

$$
\begin{aligned}
& \|\eta\|_{L^{2}\left(\Gamma_{3}\right)} \leq c_{1}\|\eta\|_{Q}, \quad \forall \eta \in Q, \\
& \|\xi\|_{L^{2}\left(\Gamma_{3}\right)} \leq c_{2}\|\xi\|_{W}, \quad \forall \xi \in W .
\end{aligned}
$$

When $q, D \in \mathcal{W}$ are sufficiently regular functions, the following Green's type formulas hold

$$
\begin{aligned}
(q, \nabla \eta)_{L^{2}(\Omega)^{d}}+(\operatorname{div} q, \eta)_{L^{2}(\Omega)} & =\int_{\Gamma} q \cdot \nu \eta d a, \quad \forall \eta \in H^{1}(\Omega), \\
(D, \nabla \xi)_{L^{2}(\Omega)^{d}}+(\operatorname{div} D, \xi)_{L^{2}(\Omega)} & =\int_{\Gamma} D \cdot n \xi d a, \quad \forall \xi \in H^{1}(\Omega) .
\end{aligned}
$$

Recall also that the transposite $\mathcal{E}^{*}$ is given by

$$
\mathcal{E} \sigma v=\sigma \mathcal{E}^{*} v, \quad \forall \sigma \in \mathbb{S}^{d} \text { and } \forall v \in \mathbb{R}^{d} .
$$

In the study of the mechanical problem (2.1)-(2.15), we need the following assumptions.

$\left(h_{1}\right)$ The elasticity operator $\mathfrak{F}: \Omega \times \mathbb{S}^{d} \rightarrow \mathbb{S}^{d}$ satisfies

$$
\begin{gathered}
\left\|\mathfrak{F}\left(x, \xi_{1}\right)-\mathfrak{F}\left(x, \xi_{2}\right)\right\| \leq M_{\mathfrak{F}}\left\|\xi_{1}-\xi_{2}\right\|, \\
\left(\mathfrak{F}\left(x, \xi_{1}\right)-\mathfrak{F}\left(x, \xi_{2}\right)\right)\left(\xi_{1}-\xi_{2}\right) \geq m_{\mathfrak{F}}\left\|\xi_{1}-\xi_{2}\right\|^{2},
\end{gathered}
$$

for all $\xi_{1}, \xi_{2} \in \mathbb{S}^{d}$ and $x \in \Omega$, where $m_{\mathfrak{F}}$ and $M_{\mathfrak{F}}$ are positive constants.

The mapping $x \rightarrow \mathfrak{F}(x, \xi)$ is measurable on $\Omega$ for all $\xi \in \mathbb{S}^{d}$ and belongs to $\mathcal{H}$ for $\xi=0$. 
$\left(h_{2}\right)$ The piezoelectric tensor $\mathcal{E}: \Omega \times \mathbb{S}^{d} \rightarrow \mathbb{R}^{d}$, the thermal expansion tensor $\mathcal{M}: \Omega \times \mathbb{R}^{d} \rightarrow \mathbb{R}^{d}$ and the pyroelectric tensor $\mathcal{P}: \Omega \rightarrow \mathbb{R}^{d}$ satisfy

$$
e_{i j k}=e_{i k j} \in L^{\infty}(\Omega), m_{i j}=m_{j i} \in L^{\infty}(\Omega), p_{i} \in L^{\infty}(\Omega) .
$$

Notice that the above conditions allows us to define

$$
M_{\mathcal{M}}=\sup _{i j}\left\|m_{i j}\right\|_{L^{\infty}(\Omega)} \text { and } M_{\mathcal{P}}=\sup _{i}\left\|p_{i}\right\|_{L^{\infty}(\Omega)} \text {. }
$$

$\left(h_{3}\right)$ The electric permittivity tensor $\beta: \Omega \times \mathbb{R}^{d} \rightarrow \mathbb{R}^{d}$ and the thermal conductivity tensor $\mathcal{K}: \Omega \times \mathbb{R}^{d} \rightarrow \mathbb{R}^{d}$ satisfy

$$
\begin{aligned}
& \beta_{i j}=\beta_{j i} \in L^{\infty}(\Omega) \text { and } \beta_{i j} z_{i} z_{j} \geq m_{\beta}\|z\|^{2}, \\
& k_{i j}=k_{j i} \in L^{\infty}(\Omega) \text { and } k_{i j} z_{i} z_{j} \geq m_{\mathcal{K}}\|z\|^{2},
\end{aligned}
$$

for all $z \in \mathbb{R}^{d}$, where $m_{\beta}$ and $m_{\mathcal{K}}$ are a positive constants.

$\left(h_{4}\right)$ The coefficient of friction function $\mu: \Gamma_{3} \times \mathbb{R}_{+} \rightarrow \mathbb{R}_{+}$satisfy

$\exists M_{\mu}>0$ such that $|\mu(x, u)| \leq M_{\mu}$, for all $u \in \mathbb{R}_{+}$and $x \in \Gamma_{3}$, $x \rightarrow \mu(x, u)$ is measurable on $\Gamma_{3}$ for all $u \in \mathbb{R}_{+}$.

$\left(h_{5}\right)$ The surface electrical conductivity $\psi: \Gamma_{3} \times \mathbb{R} \rightarrow \mathbb{R}_{+}$and the thermal conductance $k_{c}: \Gamma_{3} \times \mathbb{R} \rightarrow \mathbb{R}_{+}$satisfy for $\pi=\psi$ or $k_{c}$

$\exists M_{\pi}>0$ such that $|\pi(x, u)| \leq M_{\pi}$, for all $u \in \mathbb{R}$ and $x \in \Gamma_{3}$, $x \rightarrow \pi(x, u)$ is measurable on $\Gamma_{3}$ for all $u \in \mathbb{R}$, $\pi(x, u)=0$ for all $x \in \Gamma_{3}$ and $u \leq 0$.

$\left(h_{6}\right)$ The functions $u \rightarrow \pi(x, u)$ for $\pi=\mu\left(\right.$ resp. $\left.\pi=\psi, k_{c}\right)$ are a Lipschitz functions on $\mathbb{R}_{+}($resp. $\mathbb{R})$; for all $x \in \Gamma_{3}$ and $u_{1}, u_{2} \in \mathbb{R}$,

$$
\exists L_{\pi}>0 \text { such that }\left|\pi\left(x, u_{1}\right)-\pi\left(x, u_{2}\right)\right| \leq L_{\pi}\left|u_{1}-u_{2}\right| \text {. }
$$

$\left(h_{7}\right)$ The forces, the traction, the volume, surface charge densities, the volume heat source, the potential and temperature of the contact surface satisfy

$$
\begin{gathered}
f_{0} \in L^{2}(\Omega)^{d}, f_{2} \in L^{2}\left(\Gamma_{3}\right)^{d}, q_{0} \in L^{2}(\Omega), q_{2} \in L^{2}\left(\Gamma_{b}\right), \\
q_{t h} \in L^{2}(\Omega), \varphi_{F} \in L^{2}\left(\Gamma_{3}\right), \theta_{F} \in L^{2}\left(\Gamma_{3}\right) .
\end{gathered}
$$

$\left(h_{8}\right)$ The mapping $R: H_{\Gamma_{3}}^{\prime} \rightarrow L^{\infty}\left(\Gamma_{3}\right)$ is a linear and continuous.

Next, we use the Riesz's representation theorem to define the elements $f \in V, q \in W$ by

$$
\begin{aligned}
& (f, v)_{V}=\int_{\Omega} f_{0} v d x+\int_{\Gamma_{2}} f_{2} v d a, \forall v \in V \\
& (q, \xi)_{W}=\int_{\Omega} q_{0} \xi d x-\int_{\Gamma_{b}} q_{2} \xi d a, \forall \xi \in W
\end{aligned}
$$

and we define the mappings

$$
j: V \times V \rightarrow \mathbb{R}, \quad l: V \times W \times W \rightarrow \mathbb{R}, \quad \chi: V \times Q \times Q \rightarrow \mathbb{R},
$$

respectively, given by

$$
j(u, v)=\int_{\Gamma_{3}} \mu\left(\left\|u_{\tau}\right\|\right)\left|R \sigma_{n}(u)\right|\left\|v_{\tau}\right\| d a, \quad \forall u, v \in V,
$$




$$
\begin{aligned}
& l(u, \varphi, \xi)=\int_{\Gamma_{3}} \psi\left(u_{n}\right) \phi_{L}\left(\varphi-\varphi_{F}\right) \xi d a, \quad \forall \varphi, \xi \in W, \\
& \chi(u, \theta, \eta)=\int_{\Gamma_{3}} k_{c}\left(u_{n}\right) \phi_{L}\left(\theta-\theta_{f}\right) \eta d a, \quad \forall \theta, \eta \in Q .
\end{aligned}
$$

It follows from the assumptions $\left(h_{4}\right)$ and $\left(h_{5}\right)$ that the above integrals are welldefined. According to this notation and by a standard procedure based on Green's formulas, we can deduce the variational formulation of the problem $P$.

Problem $(P V):$ Find a displacement field $u \in K$, an electric potential $\varphi \in W$ and a temperature field $\theta \in Q$ such that

$$
\begin{aligned}
& (\mathfrak{F} \varepsilon(u), \varepsilon(v)-\varepsilon(u))_{\mathcal{H}}+\left(\mathcal{E}^{*} \nabla \varphi, \varepsilon(v)-\varepsilon(u)\right)_{L^{2}(\Omega)^{d}} \\
& -(\mathcal{M} \theta, \varepsilon(v)-\varepsilon(u))_{L^{2}(\Omega)^{d}}+j(u, v)-j(u, u) \\
& \geq(f, v-u)_{V}, \quad \forall v \in K, \\
& (\beta \nabla \varphi, \nabla \xi)_{L^{2}(\Omega)^{d}}-(\mathcal{E} \varepsilon(u), \nabla \xi)_{L^{2}(\Omega)^{d}}-(\mathcal{P} \theta, \nabla \xi)_{L^{2}(\Omega)^{d}} \\
& +l((u, \varphi), \xi)=(q, \xi)_{W}, \quad \forall \xi \in W, \\
& (\mathcal{K} \nabla \theta, \nabla \eta)_{L^{2}(\Omega)^{d}}+\chi((u, \theta), \eta)=\left(q_{t h}, \eta\right)_{L^{2}(\Omega)}, \quad \forall \eta \in Q .
\end{aligned}
$$

Now, we are able to state the following existence and uniqueness main result

Theorem 3.1. Assume that $\left(h_{1}\right)-\left(h_{5}\right)$ and $\left(h_{7}\right)-\left(h_{8}\right)$ hold, then

1) The problem $(P V)$ has at least one solution $(u, \varphi, \theta) \in K \times W \times Q$.

2) Under $\left(h_{6}\right)$, there exist a positive constant $L^{*}$ such that if

$$
L_{\mu}+M_{\mu}+M_{\psi}+M_{k_{c}}+L L_{\psi}+L L_{k_{c}}+\max \left(M_{\mathcal{M}}, M_{\mathcal{P}}\right)<L^{*},
$$

then the problem $(P V)$ has an unique solution.

The proof of this result will be treated in the next section.

\section{Proof of the main Result}

The proof of Theorem 3.1 is carried out in several steps, in the sequel, we define the tree closed convex sets of $L^{2}\left(\Gamma_{3}\right)$

$$
\begin{aligned}
& \mathcal{K}_{1}=\left\{z_{1} \geq 0 \in L^{2}\left(\Gamma_{3}\right),\left\|z_{1}\right\|_{L^{2}\left(\Gamma_{3}\right)} \leq k_{1}\right\}, \\
& \mathcal{K}_{2}=\left\{z_{2} \in L^{2}\left(\Gamma_{3}\right),\left\|z_{2}\right\|_{L^{2}\left(\Gamma_{3}\right)} \leq k_{2}\right\}, \\
& \mathcal{K}_{3}=\left\{z_{2} \in L^{2}\left(\Gamma_{3}\right),\left\|z_{3}\right\|_{L^{2}\left(\Gamma_{3}\right)} \leq k_{3}\right\},
\end{aligned}
$$


with $k_{1}, k_{2}$ and $k_{3}$ to be specified later and let $z=\left(z_{1}, z_{2}, z_{3}\right) \in L^{2}\left(\Gamma_{3}\right)^{3}$ to be given. We define the functions

$$
\begin{aligned}
& j_{1}(z, v)=\int_{\Gamma_{3}} z_{1}\left\|v_{\tau}\right\| d a, \quad \forall v \in K, \\
& \ell_{1}(z, \xi)=\int_{\Gamma_{3}} z_{2} \xi d a, \quad \forall \xi \in W, \\
& \chi_{1}(z, \eta)=\int_{\Gamma_{3}} z_{3} \eta d a, \quad \forall \eta \in Q .
\end{aligned}
$$

Now, we consider the following intermediate variational problem $(P V I)$. We can remark that this problem is decomposed into two separate problems, one is a thermal problem and the other is an electro-elastic problem.

Problem $(P V I):$ Find a displacement field $u(z) \in K$, an electric potential $\varphi(z) \in W$ and a temperature field $\theta(z) \in Q$ such that

$$
\begin{aligned}
& (\mathfrak{F} \varepsilon(u(z)), \varepsilon(v)-\varepsilon(u(z)))_{\mathcal{H}}+\left(\mathcal{E}^{*} \nabla \varphi(z), \varepsilon(v)-\varepsilon(u(z))\right)_{L^{2}(\Omega)^{d}} \\
& -(\mathcal{M} \theta(z), \varepsilon(v)-\varepsilon(u(z)))_{L^{2}(\Omega)^{d}}+j_{1}(z, v)-j_{1}(z, u(z)) \\
& \geq(f, v-u(z))_{V}, \quad \forall v \in K, \\
& (\beta \nabla \varphi(z), \nabla \xi)_{L^{2}(\Omega)^{d}}-(\mathcal{E} \varepsilon(u(z)), \nabla \xi)_{L^{2}(\Omega)^{d}}-(\mathcal{P} \theta(z), \nabla \xi)_{L^{2}(\Omega)^{d}} \\
& =(q, \xi)_{W}-\ell_{1}(z, \xi), \quad \forall \xi \in W, \\
& (\mathcal{K} \nabla \theta(z), \nabla \eta)_{L^{2}(\Omega)^{d}}=\left(q_{t h}, \eta\right)_{L^{2}(\Omega)}-\chi_{1}(z, \eta), \quad \forall \eta \in Q .
\end{aligned}
$$

First, we will solve the thermal problem in which the unknown is the temperature $\theta(z)$. Then, its solution will be an input data for the electro-elastic problem. The thermal problem is written as follows

$$
\left(P V I^{\theta}\right): \quad(\mathcal{K} \nabla \theta(z), \nabla \eta)_{L^{2}(\Omega)^{d}}=\left(q_{t h}, \eta\right)_{L^{2}(\Omega)}-\chi_{1}(z, \eta), \quad \forall \eta \in Q .
$$

We use the Riesz's representation theorem to define the element $q_{z} \in Q$ such that

$$
\left(q_{z}, \eta\right)_{Q}=\left(q_{t h}, \eta\right)_{L^{2}(\Omega)}-\chi_{1}(z, \eta), \quad \forall \eta \in Q
$$

and the operator $\mathcal{T}: Q \rightarrow Q$ such that

$$
(\mathcal{T} \theta(z), \eta)_{Q}=(\mathcal{K} \nabla \theta(z), \nabla \eta)_{L^{2}(\Omega)^{d}}, \forall \eta \in Q .
$$

Thus the variational formulation of $\left(P V I^{\theta}\right)$ will be

$$
\text { Find } \theta(z) \in Q \text { such that }\left(\mathcal{T} \theta_{z}, \eta\right)_{Q}=\left(q_{z}, \eta\right)_{Q}, \forall \eta \in Q .
$$

LEMMA 4.1. Under the assumption $\left(h_{3}\right)$, the problem (4.8) has an unique solution $\theta(z)$, which depends Lipchitz continuously on $z \in L^{2}\left(\Gamma_{3}\right)^{3}$. 
Proof. It follows from the assumptions of $\mathcal{K}\left(\right.$ see $\left.\left(h_{3}\right)\right)$ that $\mathcal{T}$ is a linear symmetric and positive definite operator. Hence, $\mathcal{T}$ is a linear continuous and invertible operator on $Q$ and let $\mathcal{C}$ denote its inverse. Thus, by the LaxMilgram theorem, we conclude that the problem (4.8) has an unique solution

$$
\theta(z)=\mathcal{C} q_{z} \in Q \quad \text { satisfying } \quad\|\theta(z)\|_{Q} \leq m_{\mathcal{C}}\left\|q_{z}\right\|_{Q},
$$

with $m_{\mathcal{C}}=\frac{1}{m_{\mathcal{K}}}$.

Let $z=\left(z_{i}\right)_{i}, z^{\prime}=\left(z_{i}^{\prime}\right)_{i}$ to be two given elements of $L^{2}\left(\Gamma_{3}\right)^{3}$ and $\theta(z)$, $\theta\left(z^{\prime}\right)$ the corresponding solutions of the problem (4.8). From the linearity of $\mathcal{T}$ we deduce that

$$
\left\|\theta\left(z^{\prime}\right)-\theta(z)\right\|_{Q} \leq m_{\mathcal{C}}\left\|q_{z^{\prime}}-q_{z}\right\|_{Q} .
$$

From (4.7) and (3.5) we obtain

$$
\left(q_{z^{\prime}}-q_{z}, \eta\right)_{Q}=\chi_{1}\left(z^{\prime}, \eta\right)-\chi_{1}(z, \eta) \leq c_{1}\left\|z_{3}^{\prime}-z_{3}\right\|_{L^{2}\left(\Gamma_{3}\right)}\|\eta\|_{Q}, \quad \forall \eta \in Q
$$

then

$$
\left\|q_{z_{1}}-q_{z_{2}}\right\|_{Q} \leq c_{1}\left\|z_{3}^{\prime}-z_{3}\right\|_{L^{2}\left(\Gamma_{3}\right)} .
$$

Finally, combine the previous inequalities to get

$$
\left\|\theta_{z_{2}}-\theta_{z_{1}}\right\|_{Q} \leq c_{1} m_{\mathcal{C}}\left\|z_{3}^{\prime}-z_{3}\right\|_{L^{2}\left(\Gamma_{3}\right)} \leq c_{1} m_{\mathcal{C}}\left\|z^{\prime}-z\right\|_{L^{2}\left(\Gamma_{3}\right)^{3}},
$$

which finishes the proof.

For the electro-elastic problem $\left(P V I^{u \varphi}\right)$, we use the solution $\theta(z)=\mathcal{C} q_{z}$ as input data and its variational formulation becomes

$$
\begin{aligned}
& (\mathfrak{F} \varepsilon(u(z)), \varepsilon(v)-\varepsilon(u(z)))_{\mathcal{H}}+\left(\mathcal{E}^{*} \nabla \varphi(z), \varepsilon(v)-\varepsilon(u(z))\right)_{L^{2}(\Omega)^{d}} \\
& +j_{1}(z, v)-j_{1}(z, u(z)) \\
& \geq(f, v-u(z))_{V}+\left(\mathcal{M C} q_{z}, \varepsilon(v)-\varepsilon(u(z))\right)_{L^{2}(\Omega)^{d}}, \quad \forall v \in K, \\
& (\beta \nabla \varphi(z), \nabla \xi)_{L^{2}(\Omega)^{d}}-(\mathcal{E} \varepsilon(u(z)), \nabla \xi)_{L^{2}(\Omega)^{d}} \\
& =(q, \xi)_{W}+\left(\mathcal{P C} q_{z}, \nabla \xi\right)_{L^{2}(\Omega)^{d}}-\ell_{1}(z, \xi), \quad \forall \xi \in W .
\end{aligned}
$$

Notice that the two facts, $\mathcal{T}$ is linear continuous invertible operator on $Q$ and the properties of the operators $\mathcal{M}$ and $\mathcal{P}$ imply that $\mathcal{M C}$ and $\mathcal{P C}$ are linear continuous operators. Hence, we use the Riesz's representation theorem to define the elements $f_{z} \in V$ and $q_{z} \in W$ such that

$$
\begin{aligned}
& \left(f_{z}, v\right)_{V}=(f, v)_{V}+\left(\mathcal{M C} q_{z}, \varepsilon(v)\right)_{L^{2}(\Omega)^{d}}, \quad \forall v \in K \\
& \left(q_{z}, \xi\right)_{W}=(q, \xi)_{W}+\left(\mathcal{P C} q_{z}, \nabla \xi\right)_{L^{2}(\Omega)^{d}}-\ell_{1}(z, \xi), \quad \forall \xi \in W .
\end{aligned}
$$


In the sequel, we consider the product spaces $X=V \times W$ and $Y=\left(L^{2}\left(\Gamma_{3}\right)\right)^{3}$ endowed with theirs canonical inner products and the associated norms

$$
\begin{array}{ll}
(x, y)_{X}=(u, v)_{V}+(\varphi, \xi)_{W}, & \forall x=(u, \varphi), y=(v, \xi) \in X, \\
(z, \zeta)_{Y}=\sum_{i=1}^{3}\left(z_{i}, \zeta_{i}\right)_{L^{2}\left(\Gamma_{3}\right)}, & \forall z=\left(z_{i}\right)_{i}, \zeta=\left(\zeta_{i}\right)_{i} \in Y
\end{array}
$$

and $U=K \times W$ a nonempty closed convex subset of $X$.

We also consider the operator $A: U \rightarrow X$, the function $\widetilde{j}_{1}(z,):. U \rightarrow \mathbb{R}$ defined as follows

$$
\begin{aligned}
(A x, y)_{X}= & (\mathfrak{F} \varepsilon(u), \varepsilon(v))_{\mathcal{H}}+(\beta \nabla \varphi, \nabla \xi)_{L^{2}(\Omega)^{d}} \\
& -\left(\mathcal{E}^{*} \nabla \varphi, \varepsilon(v)\right)_{L^{2}(\Omega)^{d}}-\left(\mathcal{E} \varepsilon\left(u_{z}\right), \nabla \xi\right)_{L^{2}(\Omega)^{d}}, \\
\widetilde{j}_{1}(z, y)= & j_{1}(z, v), \quad \forall y=(v, \xi) \in U
\end{aligned}
$$

and the element

$$
\widetilde{f}_{z}=\left(f_{z}, q_{z}\right) \in X
$$

According to these notations, we have the following equivalence result

Lemma 4.2. The pair $x(z)=(u(z), \varphi(z)) \in U$ is a solution to problem $\left(P V I^{u \varphi}\right)$ if and only if

$$
(A x(z), y-x(z))_{X}-\widetilde{j}_{1}(z, y)-\widetilde{j}_{1}(z, x(z)) \geq\left(\widetilde{f}_{z}, y-x(z)\right)_{X} .
$$

Proof. let $x(z)=(u(z), \varphi(z)) \in U$ be a solution of $\left(P V I^{u \varphi}\right)$ and $y=$ $(v, \xi) \in U$. We use $(\xi-\varphi(z))$ in $(4.11)$ and add the corresponding inequality to (4.10), we deduce (4.18). Conversely, let $x(z)=(u(z), \varphi(z)) \in U$ be a solution of the elliptic variational inequality (4.18). By taking $y=(v, \varphi(z))$ in (4.18), where $v$ is an arbitrary element of $K$, we obtain (4.10). Moreover, if we take successively $y=(u(z), \varphi(z)+\xi)$ and $y=(u(z), \varphi(z)-\xi)$ in (4.18), where $\xi$ is an arbitrary element of $W$, we will obtain (4.11), which finishes the proof. result.

We use now Lemma 4.2 to obtain the following existence and uniqueness

Lemma 4.3. For any $z \in \mathcal{K}_{1} \times \mathcal{K}_{2} \times \mathcal{K}_{3}$, assumed to be known, we have

1. Under the assumptions $\left(h_{1}\right)$ and $\left(h_{3}\right)$, the electro-elastic problem $\left(P V I^{u \varphi}\right)$ has an unique solution $x(z)=(u(z), \varphi(z)) \in K \times W$ and we have

$\exists c>0$ such that $\|x(z)\|_{X} \leq c\left\|\widetilde{f}_{z}\right\|_{X}$.

2. The solution $x(z)$ of $\left(P V I^{u \varphi}\right)$ depends Lipschitz continuously on $z$.

Proof. First, we can easily check that the operator $A: X \rightarrow X$ is strongly monotone, Lipschitz continuous and the functional $v \rightarrow \widetilde{j}_{1}(z, v)$ is proper, convex and continuous (see [6]). Therefore, the problem $\left(P V I^{u \varphi}\right)$ has 
an unique solution $x(z) \in K \times W$. Moreover, if we take $y=0$ in (4.18), we get

$$
(A x(z), x(z))_{X}+\widetilde{j}_{1}(z, x(z)) \leq\left(\widetilde{f}_{z}, x(z)\right)_{X} .
$$

As $z \in \mathcal{K}_{1} \times \mathcal{K}_{2} \times \mathcal{K}_{3}$, we have $z_{1} \geq 0$ and then $\widetilde{j}_{1}(z, x(z)) \geq 0$. Thus

$$
(A x(z), x(z))_{X} \leq\left(\widetilde{f}_{z}, x(z)\right)_{X} .
$$

Keeping in mind the strong monotony of $A$, we deduce that there exists a positive constant $c=\frac{1}{m}$ such that

$$
\|x(z)\|_{X} \leq c\left\|\tilde{f}_{z}\right\|_{X}
$$

For the second part of the lemma (4.2), let us consider $z=\left(z_{i}\right)_{i}, z^{\prime}=\left(z_{i}^{\prime}\right)_{i}$ two given elements of $L^{2}\left(\Gamma_{3}\right)^{3}$ and $x(z), x\left(z^{\prime}\right)$ theirs corresponding solutions of the problem (4.18). We have

$$
\begin{aligned}
& (A x(z), y-x(z))_{X}+\widetilde{j}_{1}(z, y)-\widetilde{j}_{1}(z, x(z)) \geq\left(\widetilde{f}_{z}, y-x(z)\right)_{X}, \\
& \left(A x\left(z^{\prime}\right), y-x\left(z^{\prime}\right)\right)_{X}+\widetilde{j}_{1}\left(z^{\prime}, y\right)-\widetilde{j}_{1}\left(z^{\prime}, x\left(z^{\prime}\right)\right) \geq\left(\widetilde{f}_{z^{\prime}}, y-x\left(z^{\prime}\right)\right)_{X}, \quad \forall y \in U .
\end{aligned}
$$

We take $y=x\left(z^{\prime}\right)$ in the first inequality and $y=x(z)$ in the second, to get

$$
\begin{aligned}
& \left(A x(z), x\left(z^{\prime}\right)-x(z)\right)_{X}+\widetilde{j}_{1}\left(z, x\left(z^{\prime}\right)\right)-\widetilde{j}_{1}(z, x(z)) \geq\left(\widetilde{f}_{z}, x\left(z^{\prime}\right)-x(z)\right)_{X}, \\
& \left(A x\left(z^{\prime}\right), x(z)-x\left(z^{\prime}\right)\right)_{X}+\widetilde{j}_{1}\left(z^{\prime}, x(z)\right)-\widetilde{j}_{1}\left(z^{\prime}, x\left(z^{\prime}\right)\right) \geq\left(\widetilde{f}_{z^{\prime}}, x(z)-x\left(z^{\prime}\right)\right)_{X} .
\end{aligned}
$$

Keeping in mind the definition of $\widetilde{j}_{1}$, we deduce

$$
\begin{aligned}
& \left(A x(z)-A x\left(z^{\prime}\right), x(z)-x\left(z^{\prime}\right)\right)_{X} \\
& \leq\left(\widetilde{f}_{z}-\widetilde{f}_{z^{\prime}}, x(z)-x\left(z^{\prime}\right)\right)_{X}+\int_{\Gamma_{3}}\left(z_{1}-z_{1}^{\prime}\right)\left(\left\|u_{\tau}(z)\right\|-\left\|u_{\tau}\left(z^{\prime}\right)\right\|\right) d a .
\end{aligned}
$$

It follows from (4.12), (4.13) that

$$
\begin{aligned}
&\left(\widetilde{f}_{z}-\widetilde{f}_{z^{\prime}}, x(z)-x\left(z^{\prime}\right)\right)_{X} \\
&=\int_{\Gamma_{3}}\left(z_{2}-z_{2}^{\prime}\right)\left(\varphi(z)-\varphi\left(z^{\prime}\right)\right) d a \\
& \quad+\left(\mathcal{M C}\left(q_{z}-q_{z^{\prime}}\right), \varepsilon(u(z))-\varepsilon\left(u\left(z^{\prime}\right)\right)\right)_{L^{2}(\Omega)^{d}} \\
& \quad+\left(\mathcal{P C}\left(q_{z}-q_{z^{\prime}}\right), \nabla \varphi(z)-\nabla \varphi\left(z^{\prime}\right)\right)_{L^{2}(\Omega)^{d}} \\
& \leq\left\|z_{2}-z_{2}^{\prime}\right\|_{L^{2}\left(\Gamma_{3}\right)}\left\|\varphi(z)-\varphi\left(z^{\prime}\right)\right\|_{L^{2}\left(\Gamma_{3}\right)} \\
&+m_{\mathcal{C}} M_{\mathcal{M}}\left\|q_{z}-q_{z^{\prime}}\right\|_{L^{2}(\Omega)}\left\|\varepsilon(u(z))-\varepsilon\left(u\left(z^{\prime}\right)\right)\right\|_{L^{2}(\Omega)^{d}} \\
&\left.+m_{\mathcal{C}} M_{\mathcal{P}}\left\|q_{z}-q_{z^{\prime}}\right\|_{L^{2}(\Omega)} \| \nabla \varphi(z)-\nabla \varphi\left(z^{\prime}\right)\right) \|_{L^{2}(\Omega)}
\end{aligned}
$$


and from (4.7) that

$$
\begin{aligned}
\left(\widetilde{f}_{z}-\right. & \left.\widetilde{f}_{z^{\prime}}, x(z)-x\left(z^{\prime}\right)\right)_{X} \\
\leq & \left\|z_{2}-z_{2}^{\prime}\right\|_{L^{2}\left(\Gamma_{3}\right)}\left\|\varphi(z)-\varphi\left(z^{\prime}\right)\right\|_{L^{2}\left(\Gamma_{3}\right)} \\
& +c_{1} m_{\mathcal{C}} M_{\mathcal{M}}\left\|z_{3}-z_{3}^{\prime}\right\|_{L^{2}(\Omega)}\left\|\varepsilon(u(z))-\varepsilon\left(u\left(z^{\prime}\right)\right)\right\|_{L^{2}(\Omega)^{d}} \\
& \left.+c_{1} m_{\mathcal{C}} M_{\mathcal{P}}\left\|z_{3}-z_{3}^{\prime}\right\|_{L^{2}(\Omega)} \| \nabla \varphi(z)-\nabla \varphi\left(z^{\prime}\right)\right) \|_{L^{2}(\Omega)} .
\end{aligned}
$$

Combining (4.20), (4.21) and (3.4), (3.6) to get

$$
\begin{aligned}
(A x(z) & \left.-A x\left(z^{\prime}\right), x(z)-x\left(z^{\prime}\right)\right)_{X} \\
\leq & c_{0}\left\|z_{1}-z_{1}^{\prime}\right\|_{L^{2}\left(\Gamma_{3}\right)}\left\|u(z)-u\left(z^{\prime}\right)\right\|_{V} \\
& +c_{2}\left\|z_{2}-z_{2}^{\prime}\right\|_{L^{2}\left(\Gamma_{3}\right)}\left\|\varphi(z)-\varphi\left(z^{\prime}\right)\right\|_{W} \\
& +c_{1} m_{\mathcal{C}} M_{\mathcal{M}}\left\|z_{3}-z_{3}^{\prime}\right\|_{L^{2}\left(\Gamma_{3}\right)}\left\|u(z)-u\left(z^{\prime}\right)\right\|_{V} \\
& \left.+c_{1} m_{\mathcal{C}} M_{\mathcal{P}}\left\|z_{3}-z_{3}^{\prime}\right\|_{L^{2}\left(\Gamma_{3}\right)} \| \varphi(z)-\varphi\left(z^{\prime}\right)\right) \|_{W} .
\end{aligned}
$$

The above inequality, the strong monotony of $A$, combined with (4.21) and (4.15) assures that there exists a positive constant $c_{3}>0$ such that

$$
\left\|x(z)-x\left(z^{\prime}\right)\right\|_{X} \leq c_{3}\left\|z-z^{\prime}\right\|_{Y} .
$$

Hence the second part of this lemma is established.

Lemma 4.4. For any $z \in \mathcal{K}_{1} \times \mathcal{K}_{2} \times \mathcal{K}_{3}$, assumed to be known and under the assumptions $\left(h_{1}\right)-\left(h_{3}\right)$, the solution $\widetilde{x}(z)=(x(z), \varphi(z), \theta(z))$ of the problem $(P V I)$ depends Lipschitz continuously on $z$.

Proof. It is a direct result of the two lemmas (4.1) and (4.3).

Now, we consider the following operator $\Lambda: Y \rightarrow Y$ defined by

$$
\Lambda z=\left(\mu\left(\left\|u_{\tau}\right\|\right)\left|R \sigma_{n}(u)\right|, \psi\left(u_{n}\right) \phi_{L}\left(\varphi-\varphi_{F}\right), k_{c}\left(u_{n}\right) \phi_{L}\left(\theta-\theta_{F}\right)\right)
$$

and notice that under the assumptions $\left(h_{5}\right)$ and $\left(h_{6}\right)$, this operator is well defined. Our aim is to prove that $\Lambda$ has a fixed point and for that we need some auxiliary lemmas.

LEMma 4.5. The mapping $z \rightarrow \widetilde{x}(z)$, where $\widetilde{x}(z)$ is the solution of the problem $(P V I)$, is weakly continuous from $Y=L^{2}\left(\Gamma_{3}\right)^{3}$ to $V \times W \times Q$.

Proof. Recall that $\widetilde{x}(z)=(u(z), \varphi(z), \theta(z))$ is a solution of $(P V I)$, means that $\theta(z)$ is a solution of $\left(P V I^{\theta}\right)$ and that $x(z)=(u(z), \varphi(z))$ is a solution of $\left(P V I^{u \varphi}\right)$. Therefore, it suffices to prove that the mapping $z \rightarrow \theta(z)$ (resp. $z \rightarrow x(z)$ ) is weakly continuous from $Y$ to $Q$ (resp. $Y$ to $V \times W$ ).

Let $z_{n}=\left(z_{n_{1}}, z_{n_{2}}, z_{n_{3}}\right)$ be a sequence of $Y$ converging weakly to $z=$ $\left(z_{1}, z_{2}, z_{3}\right)$ and let $\widetilde{x}\left(z_{n}\right)=\left(x\left(z_{n}\right), \theta\left(z_{n}\right)\right) \in U \times Q$ be its associated solution of the (PVI). From (4.9) and (4.7),(3.5) we can deduce

$$
\begin{aligned}
\left\|\theta\left(z_{n}\right)\right\|_{Q} & \leq m_{\mathcal{C}}\left\|q_{z_{n}}\right\|_{Q} \leq m_{\mathcal{C}}\left(\left\|q_{t h}\right\|_{Q}+c_{1}\left\|z_{n_{3}}\right\|_{L^{2}\left(\Gamma_{3}\right)}\right) \\
& \leq m_{\mathcal{C}}\left(\left\|q_{t h}\right\|_{Q}+c_{1}\left\|z_{n}\right\|_{Y}\right) .
\end{aligned}
$$


So, the sequence $\left(\theta\left(z_{n}\right)\right)$ is bounded in $Q$ and then, there exist $\widetilde{\theta} \in Q$ and a subsequence $\left(\theta\left(z_{n_{k}}\right)\right)$ such that $\theta\left(z_{n_{k}}\right)$ converge weakly to $\widetilde{\theta}$. By (4.8) we get

$$
(\mathcal{T} \widetilde{\theta}, \widetilde{\theta}-\eta) \leq \varliminf_{n \rightarrow \infty}\left(\mathcal{T} \theta\left(z_{n_{k}}\right), \theta\left(z_{n_{k}}\right)-\eta\right) \leq\left(q_{z}, \widetilde{\theta}-\eta\right)_{Q}
$$

Taking $\eta=\widetilde{\theta} \pm \eta^{*}$ in the previous inequality, we find

$$
\left(\mathcal{T} \widetilde{\theta}, \eta^{*}\right)=\left(q_{z}, \eta^{*}\right)_{Q}, \quad \forall \eta^{*} \in Q .
$$

According to (4.8) and (4.24), we conclude that $\widetilde{\theta}$ is a solution of $\left(P V I^{\theta}\right)$ and by the uniqueness of the solution of this variational equality we deduce that $\widetilde{\theta}=\theta(z)$. Since $\theta(z)$ is the unique limit of any subsequence $\left(\theta\left(z_{n_{k}}\right)\right)$, we deduce that the whole sequence $\left(\theta\left(z_{n}\right)\right)$ is weakly convergent to $\theta(z)$ in $Q$ and it leads to the weak continuity of $z \rightarrow \theta(z)$ from $Y$ to $Q$.

On another side, we recall that $x\left(z_{n}\right)$ is a solution of $\left(P V I^{u \varphi}\right)$ means

$$
\left(A x\left(z_{n}\right), y-x\left(z_{n}\right)\right)_{X}+\widetilde{j}_{1}\left(z_{n}, y\right)-\widetilde{j}_{1}\left(z_{n}, x\left(z_{n}\right)\right) \geq\left(\widetilde{f}_{z_{n}}, y-x\left(z_{n}\right)\right)_{X} .
$$

and if we take $y=0$, we get

$$
\left(A x\left(z_{n}\right), x\left(z_{n}\right)\right)_{X} \leq\left(\widetilde{f}_{z_{n}}, x\left(z_{n}\right)\right)_{X}-\widetilde{j}_{1}\left(z_{n}, x\left(z_{n}\right)\right) .
$$

From the definition of the element $\widetilde{f}$, we deduce

$$
\begin{aligned}
\left(\tilde{f}_{z_{n}}, x\left(z_{n}\right)\right)_{X}= & \left(f, u\left(z_{n}\right)\right)_{V}+\left(q, \varphi\left(z_{n}\right)\right)_{W}-\ell_{1}\left(z_{n}, \varphi\left(z_{n}\right)\right) \\
& +\left(\mathcal{M C} q_{z_{n}}, \varepsilon\left(u\left(z_{n}\right)\right)\right)_{L^{2}(\Omega)^{d}}+\left(\mathcal{P C} q_{z_{n}}, \nabla \varphi\left(z_{n}\right)\right)_{L^{2}(\Omega)^{d}},
\end{aligned}
$$

from (3.6) and (4.13), it exists $c_{4}=m_{\mathcal{C}}\left(M_{\mathcal{M}}+M_{\mathcal{P}}\right)>0$ such that

$$
\left\|\widetilde{f}_{z_{n}}\right\|_{X} \leq\|f\|_{V}+\|q\|_{W}+c_{2}\left\|z_{n_{2}}\right\|_{L^{2}\left(\Gamma_{3}\right)}+c_{4}\left\|q_{z_{n}}\right\|_{Q}
$$

from (3.5) and (4.7), we have

$$
\left\|q_{z_{n}}\right\|_{Q} \leq\left\|q_{t h}\right\|_{Q}+c_{1}\left\|z_{n_{3}}\right\|_{L^{2}\left(\Gamma_{3}\right)}
$$

and from the definition of the functional $\widetilde{j}_{1}$, we have

$$
\widetilde{j}_{1}\left(z_{n}, x\left(z_{n}\right)\right) \leq c_{0}\left\|z_{n_{1}}\right\|_{L^{2}\left(\Gamma_{3}\right)}\left\|x\left(z_{n}\right)\right\|_{X} .
$$

The strong monotony of $A$, combined with (4.26)-(4.29), leads to

$$
\begin{aligned}
m\left\|x\left(z_{n}\right)\right\|_{X} \leq & \|f\|_{V}+\|q\|_{W}+c_{4}\left\|q_{t h}\right\|_{Q}+c_{0}\left\|z_{n_{1}}\right\|_{L^{2}\left(\Gamma_{3}\right)} \\
& +c_{2}\left\|z_{n_{2}}\right\|_{L^{2}\left(\Gamma_{3}\right)}+c_{1} c_{4}\left\|z_{n_{3}}\right\|_{L^{2}\left(\Gamma_{3}\right)} \\
\leq & \|f\|_{V}+\|q\|_{W}+c_{4}\left\|q_{t h}\right\|_{Q} \\
& +c_{5}\left(\left\|z_{n_{1}}\right\|_{L^{2}\left(\Gamma_{3}\right)}+\left\|z_{n_{2}}\right\|_{L^{2}\left(\Gamma_{3}\right)}+\left\|z_{n_{3}}\right\|_{L^{2}\left(\Gamma_{3}\right)}\right),
\end{aligned}
$$

where $c_{5}=\max \left(c_{0}, c_{2}, c_{1} c_{4}\right)$.

Applying the inequality $(a+b+c) \leq \sqrt{3} \sqrt{a^{2}+b^{2}+c^{2}}$ to get

$$
\left\|x\left(z_{n}\right)\right\|_{X} \leq \frac{1}{m}\left\{\|f\|_{V}+\|q\|_{W}+c_{4}\left\|q_{t h}\right\|_{Q}+\sqrt{3} c_{5}\left\|z_{n}\right\|_{Y}\right\} .
$$


Thus, the sequence $\left(x\left(z_{n}\right)\right)$ is bounded in the Hilbert space $X$, then there exists $\widetilde{x}=(\widetilde{u}, \widetilde{\varphi}) \in X$ and a subsequence $\left(x\left(z_{n_{k}}\right)\right)$ such that $x\left(z_{n_{k}}\right) \rightarrow \widetilde{x}$. Since $U \subset X$ is closed convex subset, it is weakly closed and hence $\widetilde{x} \in U$. Moreover, by using the compactness of the trace map $\gamma: X \rightarrow L^{2}\left(\Gamma_{3}\right)^{d} \times L^{2}\left(\Gamma_{3}\right)$, it follows from the weak convergence of $\left(x\left(z_{n_{k}}\right)\right)$ that

$$
\left(x\left(z_{n_{k}}\right)\right) \rightarrow \widetilde{x} \text { strongly in } L^{2}\left(\Gamma_{3}\right)^{d} \times L^{2}\left(\Gamma_{3}\right) .
$$

Next, let us prove that $\widetilde{x}$ is solution of (4.29). We have

$$
\begin{aligned}
\left(\widetilde{f}_{z_{n}}, y-x\left(z_{n}\right)\right)_{X}= & \left(f, v-u\left(z_{n}\right)\right)_{V}+\left(q, \xi-\varphi\left(z_{n}\right)\right)_{W}-\ell_{1}\left(z_{n}, \xi-\varphi\left(z_{n}\right)\right) \\
& +\left(\mathcal{M C} q_{z_{n}}, \varepsilon(v)-\varepsilon\left(u\left(z_{n}\right)\right)\right)_{L^{2}(\Omega)^{d}} \\
& +\left(\mathcal{P C} q_{z_{n}}, \nabla \xi-\nabla \varphi\left(z_{n}\right)\right)_{L^{2}(\Omega)^{d}} .
\end{aligned}
$$

From (4.3), (4.7) and the weak convergence of $\left(z_{n}\right)$ to $z$, we get

$$
\begin{aligned}
\left(\mathcal{M C} q_{z_{n}}, \varepsilon(v)-\varepsilon\left(u\left(z_{n}\right)\right)\right)_{L^{2}(\Omega)^{d}} & \rightarrow\left(\mathcal{M C} q_{z}, \varepsilon(v)-\varepsilon(u(z))\right)_{L^{2}(\Omega)^{d}} \\
\left(\mathcal{P C} q_{z_{n}}, \nabla \xi-\nabla \varphi\left(z_{n}\right)\right)_{L^{2}(\Omega)^{d}} & \rightarrow\left(\mathcal{P C} q_{z}, \nabla \xi-\nabla \varphi(z)\right)_{L^{2}(\Omega)^{d}}
\end{aligned}
$$

from the definition of $\ell_{1}$, we get

$$
\begin{aligned}
\left|\ell_{1}\left(z_{n}, \xi-\varphi_{n}\right)-\ell_{1}\left(z_{n}, \xi-\widetilde{\varphi}\right)\right| & \leq\left\|z_{n_{2}}\right\|_{L^{2}\left(\Gamma_{3}\right)}\left\|\varphi\left(z_{n}\right)-\widetilde{\varphi}\right\|_{L^{2}\left(\Gamma_{3}\right)} \\
& \leq\left\|z_{n}\right\|_{L^{2}\left(\Gamma_{3}\right)^{3}}\left\|x\left(z_{n}\right)-\widetilde{x}\right\|_{L^{2}\left(\Gamma_{3}\right)^{2}},
\end{aligned}
$$

and hence, we deduce

$$
\ell_{1}\left(z_{n}, \xi-\varphi\left(z_{n}\right)\right) \rightarrow \ell_{1}(z, \xi-\widetilde{\varphi}) .
$$

The two previous results (4.32) and (4.33) give

$$
\left(\widetilde{f}_{z_{n}}, y-x\left(z_{n}\right)\right)_{X} \rightarrow\left(\widetilde{f}_{z}, y-\widetilde{x}\right)_{X} .
$$

Furthermore, the following inequality

$$
\begin{aligned}
\left|\widetilde{j}_{1}\left(z_{n}, x\left(z_{n}\right)\right)-\widetilde{j}_{1}\left(z_{n}, \widetilde{x}\right)\right| & \leq\left\|z_{n_{1}}\right\|_{L^{2}\left(\Gamma_{3}\right)}\left\|u\left(z_{n}\right)-\widetilde{u}\right\|_{L^{2}\left(\Gamma_{3}\right)^{d}} \\
& \leq c_{0}\left\|z_{n}\right\|_{L^{2}\left(\Gamma_{3}\right)^{3}}\left\|x\left(z_{n}\right)-\widetilde{x}\right\|_{L^{2}\left(\Gamma_{3}\right)^{2}}
\end{aligned}
$$

leads to

$$
\widetilde{j}_{1}\left(z_{n}, x\left(z_{n}\right)\right) \rightarrow \widetilde{j}_{1}(z, \widetilde{x}) .
$$

On the other hand, it follows from (4.25) that

$$
\begin{aligned}
\left(A x\left(z_{n}\right), x\left(z_{n}\right)-y\right)_{X} \leq & \left(\widetilde{f}_{z_{n}}, x\left(z_{n}\right)-y\right)_{X} \\
& +\left(\widetilde{j}_{1}\left(z_{n}, y\right)-\widetilde{j}_{1}(z, \widetilde{x})\right)-\left(\widetilde{j}_{1}\left(z_{n}, x\left(z_{n}\right)\right)-\widetilde{j}_{1}(z, \widetilde{x})\right) .
\end{aligned}
$$

Using (4.34) and (4.35) we deduce

$$
\varlimsup_{n \rightarrow \infty}\left(A x\left(z_{n}\right), x\left(z_{n}\right)-y\right)_{X} \leq\left(\widetilde{f}_{z}, \widetilde{x}-y\right)_{X}+\left(\widetilde{j}_{1}(z, y)-\widetilde{j}_{1}(z, \widetilde{x})\right),
$$


and, then

$$
\begin{aligned}
\varlimsup_{n \rightarrow \infty}\left(A x\left(z_{n}\right), x\left(z_{n}\right)-\widetilde{x}\right)_{X}=\varlimsup_{n \rightarrow \infty}\left\{\left(A x\left(z_{n}\right), x\left(z_{n}\right)-y\right)_{X}+\left(A x\left(z_{n}\right), y-\widetilde{x}\right)_{X}\right\} \\
\leq \varlimsup_{n \rightarrow \infty}\left\{\left(A x\left(z_{n}\right), x\left(z_{n}\right)-y\right)_{X}+\left\|A x\left(z_{n}\right)\right\|_{X}\|y-\widetilde{x}\|_{X}\right\} \\
\leq\left(\widetilde{f}_{z}, \widetilde{x}-y\right)_{X}+\left(\widetilde{j}_{1}(z, y)-\widetilde{j}_{1}(z, \widetilde{x})\right) \\
\quad+\varlimsup_{n \rightarrow \infty}\left\{\left\|A x\left(z_{n}\right)\right\|_{X}\|y-\widetilde{x}\|_{X}\right\} .
\end{aligned}
$$

Thus, for all $y \in U$, we have

$$
\begin{aligned}
\varlimsup_{n \rightarrow \infty}\left(A x\left(z_{n}\right), x\left(z_{n}\right)-\widetilde{x}\right)_{X} \leq & \left(\widetilde{f}_{z}, \widetilde{x}-y\right)_{X}+\left(\widetilde{j}_{1}(z, y)-\widetilde{j}_{1}(z, \widetilde{x})\right) \\
& +\varlimsup_{n \rightarrow \infty}\left\{\left\|A x\left(z_{n}\right)\right\|_{X}\|y-\widetilde{x}\|_{X}\right\} .
\end{aligned}
$$

Since $\left(\left\|A x\left(z_{n}\right)\right\|_{X}\right)$ is bounded, if we take $y=\widetilde{x}$, the last inequality becomes

$$
\varlimsup_{n \rightarrow \infty}\left(A x\left(z_{n}\right), x\left(z_{n}\right)-\widetilde{x}\right)_{X} \leq 0 .
$$

We combine (4.25), (4.34) and (4.36) with the pseudo-monotonicity of $A$ :

$$
(A \widetilde{x}, \widetilde{x}-y)_{X} \leq \varliminf_{n \rightarrow \infty}\left(A x\left(z_{n}\right), x\left(z_{n}\right)-y\right)_{X}, \quad \forall y \in U,
$$

to deduce

$$
\left\{\begin{array}{l}
\widetilde{x} \in U, \\
(A \widetilde{x}, y-\widetilde{x})_{X}+\widetilde{j}_{1}(z, y)-\widetilde{j}_{1}(z, \widetilde{x}) \geq\left(\widetilde{f}_{z}, y-\widetilde{x}\right)_{X}, \quad \forall y \in U .
\end{array}\right.
$$

Thus, we find that $\widetilde{x}$ is a solution of the problem $\left(P V I^{u \varphi}\right)$ and from the uniqueness of the solution for this variational inequality, we can deduce that $\widetilde{x}=x(z)$. Moreover, since $x(z)$ is the unique weak limit of any subsequence of $\left(x\left(z_{n}\right)\right)$, we obtain that the whole sequence $\left(x\left(z_{n}\right)\right)$ is weakly convergent in $X$ to $x(z)$. Consequently, the mapping $z \rightarrow x(z)$ is weakly continuous.

Lemma 4.6. For a specified values of $k_{1}, k_{2}$ and $k_{3}$, the operator $\Lambda$ has at least one fixed point.

Proof. Let us consider $z=\left(z_{1}, z_{2}, z_{3}\right)$ an element of $\mathcal{K}_{1} \times \mathcal{K}_{2} \times \mathcal{K}_{3}$, we have $\left\|z_{1}\right\|_{L^{2}\left(\Gamma_{3}\right)} \leq k_{1},\left\|z_{2}\right\|_{L^{2}\left(\Gamma_{3}\right)} \leq k_{2}$ and $\left\|z_{3}\right\|_{L^{2}\left(\Gamma_{3}\right)} \leq k_{3}$. Therefore

$$
\|z\|_{L^{2}\left(\Gamma_{3}\right)^{3}} \leq k_{1}+k_{2}+k_{3} .
$$

On another hand, since $\psi\left(u_{n}(z)\right) \leq M_{\psi}$ and $\phi_{L}\left(\varphi(z)-\varphi_{F}\right) \leq L$, it follows from $z_{2}=\psi\left(u_{n}(z)\right) \phi_{L}\left(\varphi(z)-\varphi_{F}\right)$ that

$$
\left\|z_{2}\right\|_{L^{2}\left(\Gamma_{3}\right)} \leq M_{\psi} \operatorname{Lmeas}\left(\Gamma_{3}\right)^{\frac{1}{2}} .
$$

Since $k_{c}\left(u_{n}(z)\right) \leq M_{k_{c}}$ and $\phi_{L}\left(\theta(z)-\theta_{F}\right) \leq L$, it follows from the expression $z_{3}=k_{c}\left(u_{n}(z)\right) \phi_{L}\left(\theta(z)-\theta_{F}\right)$ that

$$
\left\|z_{3}\right\|_{L^{2}\left(\Gamma_{3}\right)} \leq M_{k_{c}} \operatorname{Lmeas}\left(\Gamma_{3}\right)^{\frac{1}{2}} .
$$


Moreover, since $\mu\left(\left\|u_{\tau}(z)\right\|\right) \leq M_{\mu}$ and $R$ is linear continuous function, it follows from $z_{1}=\mu\left(\left\|u_{\tau}(z)\right\|\right)\left|R \sigma_{n}(u(z))\right|$ and (3.4) that

$$
\left\|z_{1}\right\|=\left\|\mu\left(\left\|u_{\tau}(z)\right\|\right)\left|R \sigma_{n}(u(z))\right|\right\| \leq M_{\mu} c_{0} c_{R}\|x(z)\|_{X} .
$$

Combining (4.19) and (4.27), (4.28), we deduce that

$$
\begin{aligned}
\|x(z)\|_{X} \leq & c\left\{\|f\|_{V}+\|q\|_{W}+c_{4}\left\|q_{t h}\right\|_{Q}\right\} \\
& +c\left\{c_{2}\left\|z_{2}\right\|_{L^{2}\left(\Gamma_{3}\right)}+c_{1} c_{4}\left\|z_{3}\right\|_{L^{2}\left(\Gamma_{3}\right)}\right\} .
\end{aligned}
$$

Keeping in mind (4.40) and (4.38), (4.39), then

$$
\begin{aligned}
\left\|z_{1}\right\| \leq & c c_{0} c_{R} M_{\mu}\left\{\|f\|_{V}+\|q\|_{W}+c_{4}\left\|q_{t h}\right\|_{Q}\right\} \\
& +c c_{0} c_{2} c_{R} M_{\mu} M_{\psi} \operatorname{Lmeas}\left(\Gamma_{3}\right)^{\frac{1}{2}} \\
& +c c_{0} c_{1} c_{4} c_{R} M_{\mu} M_{k_{c}} \operatorname{Lmeas}\left(\Gamma_{3}\right)^{\frac{1}{2}}
\end{aligned}
$$

From the definition of the operator $\Lambda$, we have

$\|\Lambda z\| \leq\left\|\mu\left(\left\|u_{\tau}\right\|\right)\left|R \sigma_{n}(u)\right|\right\|+\left\|\psi\left(u_{n}\right) \phi_{L}\left(\varphi-\varphi_{F}\right)\right\|+\left\|k_{c}\left(u_{n}\right) \phi_{L}\left(\theta-\theta_{f}\right)\right\|$.

Hence, if we choose

$$
\begin{gathered}
k_{1}=c c_{0} c_{R} M_{\mu}\left\{\|f\|_{V}+\|q\|_{W}+c_{4}\left\|q_{t h}\right\|_{Q}\right)+c_{2} M_{\psi} \operatorname{Lmeas}\left(\Gamma_{3}\right)^{\frac{1}{2}} \\
\left.+c_{1} c_{4} M_{k_{c}} \operatorname{Lmeas}\left(\Gamma_{3}\right)^{\frac{1}{2}}\right\}
\end{gathered}
$$

and

$$
k_{2}=M_{\psi} \operatorname{Lmeas}\left(\Gamma_{3}\right)^{\frac{1}{2}}, \quad k_{3}=M_{k_{c}} \operatorname{Lmeas}\left(\Gamma_{3}\right)^{\frac{1}{2}},
$$

we will obtain from (4.41) and (4.38), (4.39) that

$$
\|\Lambda z\| \leq k_{1}+k_{2}+k_{3} .
$$

Thus, $\Lambda$ is an operator from the nonempty, convex and closed subset $\mathcal{K}_{1} \times \mathcal{K}_{2} \times \mathcal{K}_{3}$ of $L^{2}\left(\Gamma_{3}\right)^{3}$ into itself. Since the space $L^{2}\left(\Gamma_{3}\right)^{3}$ is reflexive, $\mathcal{K}_{1} \times \mathcal{K}_{2} \times \mathcal{K}_{3}$ is weakly compact. The assumptions $\left(h_{5}\right),\left(h_{6}\right)$ and the continuity of the operators $R, \phi_{L}$, and $k_{c}$, combined with the lemma (4.5) lead to the weak continuity of $\Lambda$. Hence, by the Schauder's fixed point theorem the operator $\Lambda$ has at least one fixed point.

Now, we have all the ingredients to provide the proof of Theorem 3.1.

Existence: Let $z^{*}$ be the fixed point of the operator $\Lambda$ and let denote by $\widetilde{x}^{*}=\left(u^{*}, \varphi^{*}, \theta^{*}\right)$ the solution of the variational problem $(P V I)$ for $z=z^{*}$. The definition of $\Lambda$ and $(P V I)$ prove that $x^{*}$ is a solution of $(P V)$ and that leads to the existence part of theorem 3.1.

Uniqueness: We consider the product space $\mathcal{X}=X \times Q=V \times W \times Q$ endowed with the following inner product and the associated norm $\|\cdot\|_{\widetilde{X}}$ such that

$$
(\widetilde{x}, \widetilde{y})_{\mathcal{X}}=(x, y)_{X}+(\theta, \eta)_{Q}=(u, v)_{V}+(\varphi, \xi)_{W}+(\theta, \eta)_{Q},
$$

for all $\widetilde{x}=(x, \theta)=(u, \varphi, \theta)$ and $\widetilde{y}=(y, \eta)=(v, \xi, \eta)$ elements of $\mathcal{X}$. 
We consider also the two operators $\widetilde{A}: \mathcal{X} \rightarrow \mathcal{X}$ and $\widetilde{B}: \mathcal{X} \rightarrow \mathcal{X}$ defined by

$$
\begin{aligned}
& (\widetilde{A} \widetilde{x}, \widetilde{y})_{\mathcal{X}}=(A x, y)_{X}+(\mathcal{K} \nabla \theta, \nabla \eta)_{L^{2}(\Omega)^{d}}, \\
& (B \widetilde{x}, \widetilde{y})_{\mathcal{X}}=-(\mathcal{M} \theta, \varepsilon(v))_{L^{2}(\Omega)^{d}}-(\mathcal{P} \theta, \nabla \xi)_{L^{2}(\Omega)^{d}},
\end{aligned}
$$

where $A$ is given by (4.16) and the functionals $J, \widetilde{\ell}, \widetilde{\chi}$ are defined by

$$
J(\widetilde{x}, \widetilde{y})=j(u, v),
$$$$
\widetilde{\ell}(\widetilde{x}, \widetilde{y})=\ell(u, \varphi, \xi),
$$$$
\widetilde{\chi}(\widetilde{x}, \widetilde{y})=\chi(u, \theta, \eta)
$$

and the element $\tilde{f}$ is given by

$$
\widetilde{f}=\left(f, q, q_{t h}\right) \in \mathcal{X} .
$$

LEMma 4.7. The operator $\widetilde{A}$ is strongly monotone on $\mathcal{X}$ i.e.,

$$
\exists m_{\widetilde{A}}>0 \text { such that } m_{\widetilde{A}}\left\|\widetilde{x}_{1}-\widetilde{x}_{2}\right\|_{\mathcal{X}}^{2} \leq\left(\widetilde{A} \widetilde{x}_{1}-\widetilde{A} \widetilde{x}_{2}, \widetilde{x}_{1}-\widetilde{x}_{2}\right)_{\mathcal{X}} .
$$

Proof. Let $\widetilde{x}_{1}=\left(u_{1}, \varphi_{1}, \theta_{1}\right)$ and $\widetilde{x}_{2}=\left(u_{2}, \varphi_{2}, \theta_{2}\right)$ be two elements of $\mathcal{X}$. It follows from the definition of the operator $\widetilde{A}$ that

$\left(\widetilde{A} \widetilde{x}_{1}-\widetilde{A} \widetilde{x}_{2}, \widetilde{x}_{1}-\widetilde{x}_{2}\right)_{\mathcal{X}}=\left(A x_{1}-A x_{2}, x_{1}-x_{2}\right)_{X}+\left(\mathcal{K} \nabla\left(\theta_{1}-\theta_{2}\right), \nabla\left(\theta_{1}-\theta_{2}\right)\right)_{L^{2}(\Omega)^{d}}$.

Using the strong monotony of $A$ and the assumption $\left(h_{4}\right)$, we obtain

$$
\left(\widetilde{A} \widetilde{x}_{1}-\widetilde{A} \widetilde{x}_{2}, \widetilde{x}_{1}-\widetilde{x}_{2}\right)_{\mathcal{X}} \geq m\left\|x_{1}-x_{2}\right\|_{X}^{2}+m_{\mathcal{K}}\left\|\theta_{1}-\theta_{2}\right\|_{Q}^{2} .
$$

Keeping in mind (4.41), there exists $m_{\widetilde{A}}>0$ such that

$$
\left(\widetilde{A} \widetilde{x}_{1}-\widetilde{A} \widetilde{x}_{2}, \widetilde{x}_{1}-\widetilde{x}_{2}\right)_{\mathcal{X}} \geq m_{\widetilde{A}}\left\|\widetilde{x}_{1}-\widetilde{x}_{2}\right\|_{\mathcal{X}}^{2} .
$$

Lemma 4.8. The problem $(P V)$ is equivalent to the problem

$$
\begin{aligned}
&(\widetilde{A} \widetilde{x}, \widetilde{y}-\mathcal{X})_{\mathcal{X}}+(\widetilde{B} \widetilde{x}, \widetilde{y}-\widetilde{x})_{\mathcal{X}}+J(\widetilde{x}, \widetilde{y})-J(\widetilde{x}, \widetilde{x}) \\
&+\widetilde{\ell}(\widetilde{x}, \widetilde{y}-\widetilde{x})+\widetilde{\chi}(\widetilde{x}, \widetilde{y}-\widetilde{x}) \geq(\widetilde{f}, \widetilde{y}-\widetilde{x})_{\mathcal{X}}
\end{aligned}
$$

Proof. We use the same arguments as for (4.18).

Let $\widetilde{x}_{1}=\left(u_{1}, \varphi_{1}, \theta_{1}\right)$ and $\widetilde{x}_{2}=\left(u_{2}, \varphi_{2}, \theta_{2}\right)$ be solutions of $(P V)$, then

$$
\begin{aligned}
&\left(\widetilde{A} \widetilde{x}_{1}, \widetilde{y}-\widetilde{x}_{1}\right)_{\mathcal{X}}+\left(\widetilde{B} \widetilde{x}_{1}, \widetilde{y}-\widetilde{x}_{1}\right)_{\mathcal{X}}+J\left(\widetilde{x}_{1}, \widetilde{y}\right)-J\left(\widetilde{x}_{1}, \widetilde{x}_{1}\right) \\
&++\widetilde{\ell}\left(\widetilde{x}_{1}, \widetilde{y}-\widetilde{x}_{1}\right)+\widetilde{\chi}\left(\widetilde{x}_{1}, \widetilde{y}-\widetilde{x}_{1}\right) \geq\left(\widetilde{f}, \widetilde{y}-\widetilde{x}_{1}\right)_{\mathcal{X}}, \\
&\left(\widetilde{A} \widetilde{x}_{2}, \widetilde{y}-\widetilde{x}_{2}\right)_{\mathcal{X}}+\left(\widetilde{B} \widetilde{x}_{2}, \widetilde{y}-\widetilde{x}_{2}\right)_{\mathcal{X}}+J\left(\widetilde{x}_{2}, \widetilde{y}\right)-J\left(\widetilde{x}_{2}, \widetilde{x}_{2}\right) \\
&+\widetilde{\ell}\left(\widetilde{x}_{2}, \widetilde{y}-\widetilde{x}_{2}\right)+\widetilde{\chi}\left(\widetilde{x}_{2}, \widetilde{y}-\widetilde{x}_{2}\right) \geq\left(\widetilde{f}, \widetilde{y}-\widetilde{x}_{2}\right)_{\mathcal{X}} .
\end{aligned}
$$

If we take $\widetilde{y}=\widetilde{x}_{2}$ in the first inequality, $\widetilde{y}=\widetilde{x}_{1}$ in the second one and we add the two resulting inequalities, we get

$$
\left(\widetilde{A} \widetilde{x}_{1}-\widetilde{A} \widetilde{x}_{2}, \widetilde{x}_{1}-\widetilde{x}_{2}\right)_{\mathcal{X}} \leq G_{1}+G_{2}+G_{3}-\left(\widetilde{B} \widetilde{x}_{1}-\widetilde{B} \widetilde{x}_{2}, \widetilde{x}_{1}-\widetilde{x}_{2}\right)_{\mathcal{X}}
$$


such that

$$
\begin{aligned}
G_{1} & =J\left(\widetilde{x}_{1}, \widetilde{x}_{2}\right)-J\left(\widetilde{x}_{1}, \widetilde{x}_{1}\right)+J\left(\widetilde{x}_{2}, \widetilde{x}_{1}\right)-J\left(\widetilde{x}_{2}, \widetilde{x}_{2}\right), \\
G_{2} & =\widetilde{\ell}\left(\widetilde{x}_{1}, \widetilde{x}_{2}-\widetilde{x}_{1}\right)-\widetilde{\ell}\left(\widetilde{x}_{2}, \widetilde{x}_{1}-\widetilde{x}_{2}\right), \\
G_{3} & =\widetilde{\chi}\left(\widetilde{x}_{1}, \widetilde{x}_{2}-\widetilde{x}_{1}\right)-\widetilde{\chi}\left(\widetilde{x}_{2}, \widetilde{x}_{1}-\widetilde{x}_{2}\right) .
\end{aligned}
$$

We have

$$
\begin{aligned}
G_{1}= & \int_{\Gamma_{3}} \mu\left(\left\|u_{1 \tau}\right\|\right)\left(\left|R \sigma_{n}\left(u_{1}\right)\right|-\left|R \sigma_{n}\left(u_{2}\right)\right|\right)\left(\left\|u_{1 \tau}\right\|-\left\|u_{2 \tau}\right\|\right) d a \\
& +\int_{\Gamma_{3}}\left|R \sigma_{n}\left(u_{2}\right)\right|\left(\mu\left(\left\|u_{1 \tau}\right\|\right)-\mu\left(\left\|u_{2 \tau}\right\|\right)\right)\left(\left\|u_{1 \tau}\right\|-\left\|u_{2 \tau}\right\|\right) d a .
\end{aligned}
$$

Using the property of $\mu$, the continuity of $R$ and (3.4), we deduce

$$
G_{1} \leq\left(M_{\mu} c_{R} c_{0}^{2}+\|R\|_{L^{\infty}\left(\Gamma_{3}\right)} L_{\mu} c_{0}^{2}\right)\left\|\widetilde{x}_{1}-\widetilde{x}_{2}\right\|_{\mathcal{X}}^{2} .
$$

We have also

$$
\begin{aligned}
G_{2}= & \int_{\Gamma_{3}} \psi\left(u_{2 \tau}\right)\left(\phi_{L}\left(\varphi_{2}-\varphi_{F}\right)-\phi_{L}\left(\varphi_{1}-\varphi_{F}\right)\right)\left(\varphi_{1}-\varphi_{2}\right) d a \\
& +\int_{\Gamma_{3}} \phi_{L}\left(\varphi_{2}-\varphi_{F}\right)\left(\psi\left(u_{2 \tau}\right)-\psi\left(u_{1 \tau}\right)\right)\left(\varphi_{1}-\varphi_{2}\right) d a
\end{aligned}
$$

and

$$
\begin{aligned}
G_{3}= & \int_{\Gamma_{3}} k_{c}\left(u_{2 v}-g\right)\left(\phi_{L}\left(\theta_{2}-\theta_{F}\right)-\phi_{L}\left(\theta_{1}-\theta_{F}\right)\right)\left(\theta_{1}-\theta_{2}\right) d a \\
& +\int_{\Gamma_{3}} \phi_{L}\left(\theta_{2}-\theta_{F}\right)\left(k_{c}\left(u_{2 v}-g\right)-k_{c}\left(u_{1 v}-g\right)\right)\left(\theta_{1}-\theta_{2}\right) d a .
\end{aligned}
$$

Using the properties of $\phi_{L}, \psi, k_{c}$ we deduce

$$
\begin{aligned}
G_{2} & \leq\left(M_{\psi} c_{1}^{2}+L L_{\psi} c_{0} c_{1}\right)\left\|\widetilde{x}_{1}-\widetilde{x}_{2}\right\|_{\mathcal{X}}^{2}, \\
G_{3} & \leq\left(M_{k_{c}} c_{2}^{2}+L L_{k_{c}} c_{0} c_{2}\right)\left\|\widetilde{x}_{1}-\widetilde{x}_{2}\right\|_{\mathcal{X}}^{2} .
\end{aligned}
$$

On the other hand, it follows from (4.43) and the assumption $\left(h_{2}\right)$ that

$$
\begin{aligned}
\left|\left(B \widetilde{x}_{1}-B \widetilde{x}_{2}, \widetilde{x}_{1}-\widetilde{x}_{2}\right)_{\mathcal{X}}\right| & \leq M_{\mathcal{M}}\left\|\theta_{1}-\theta_{2}\right\|_{L^{2}(\Omega)}\left\|\varepsilon\left(u_{1}\right)-\varepsilon\left(u_{1}\right)\right\|_{L^{2}(\Omega)^{d}} \\
& +M_{\mathcal{P}}\left\|\theta_{1}-\theta_{2}\right\|_{L^{2}(\Omega)}\left\|\nabla \varphi_{1}-\nabla \varphi_{2}\right\|_{L^{2}(\Omega)^{d}} \\
\leq & \max \left(M_{\mathcal{M}}, M_{\mathcal{P}}\right)\left\|\theta_{1}-\theta_{2}\right\|_{H^{1}(\Omega)}\left(\left\|u_{1}-u_{2}\right\|_{V}+\left\|\varphi_{1}-\varphi_{2}\right\|_{W}\right) \\
\leq & \max \left(M_{\mathcal{M}}, M_{\mathcal{P}}\right)\left\|\theta_{1}-\theta_{2}\right\|_{H^{1}(\Omega)}\left\|x_{1}-x_{2}\right\|_{X} .
\end{aligned}
$$

Since the two norms $\|\cdot\|_{Q}$ and $\|\cdot\|_{H^{1}(\Omega)}$ are equivalents and since

$$
\|\cdot\|_{\mathcal{X}}^{2}=\|\cdot\|_{X}^{2}+\|\cdot\|_{Q}^{2} \geq 2\|\cdot\|_{X}\|\cdot\|_{Q},
$$

there exists a positive constant $c_{*}$ such that

$$
\left|\left(B \widetilde{x}_{1}-B \widetilde{x}_{2}, \widetilde{x}_{1}-\widetilde{x}_{2}\right)_{\mathcal{X}}\right| \leq c_{*} \max \left(M_{\mathcal{M}}, M_{\mathcal{P}}\right)\left\|\widetilde{x}_{1}-\widetilde{x}_{2}\right\|_{\mathcal{X}}^{2} .
$$


Finally, we combine (4.46) and (4.47)-(4.50) to deduce that there exists a positive constant $c^{*}$ such that

$$
\begin{aligned}
\left\|\widetilde{x}_{1}-\widetilde{x}_{2}\right\|_{\mathcal{X}}^{2} \leq & c^{*}\left(L_{\mu}+M_{\mu}+M_{\psi}+M_{k_{c}}+L L_{\psi}+L L_{k_{c}}\right. \\
& \left.+\max \left(M_{\mathcal{M}}, M_{\mathcal{P}}\right)\right)\left\|\widetilde{x}_{1}-\widetilde{x}_{2}\right\|_{\mathcal{X}}^{2} .
\end{aligned}
$$

Let $L^{*}=\frac{1}{c^{*}}$, then if we have

$$
L_{\mu}+M_{\mu}+M_{\psi}+M_{k_{c}}+L L_{\psi}+L L_{k_{c}}+\max \left(M_{\mathcal{M}}, M_{\mathcal{P}}\right)<L^{*},
$$

we obtain $\widetilde{x}_{1}=\widetilde{x}_{2}$ and it implies the uniqueness of the solution.

\section{REFERENCES}

[1] M. Aouadi, Generalized thermo-piezoelectric problems with temperature-dependent properties, Int. J. Solids and Structures 43 (2006), 6347-6358.

[2] R.C. Batra and J.S. Yang, Saint-Venant's principle in linear piezoelectricity, J. Elasticity 38 (1995), 209-218.

[3] P. Bisegna, F. Lebon and F. Maceri, The unilateral frictional contact of a piezoelectric body with a rigid support, in: Contact Mechanics, Kluwer, Dordrecht, 2002, 347-354.

[4] D.S. Chandrasekharaiah, A generalized linear thermoelasticity theory for piezoelectric media, Acta Mech. 71 (1984), 39-49.

[5] Z. Denkowski, S. Migórski and A. Ochal, A class of optimal control problems for piezoelectric frictional contact models, Nonlinear Anal. Real World Appl. 12 (2011), $1883-1895$.

[6] El H. Essoufi, El H. Benkhira and R. Fakhar, Analysis and numerical approximation of an electro-elastic frictional contact problem, Adv. Appl. Math. Mech. 2 (2010), $355-378$.

[7] T. Ikeda, Fundamentals of piezoelectricity, Oxford University Press, Oxford, 1990.

[8] S. Migórski, Hemivariational inequality for a frictional contact problem in elastopiezoelectricity, Discrete Contin. Dyn. Syst. Ser. B 6 (2006), 1339-1356.

[9] S. Migórski, A class of hemivariational inequality for electroelastic contact problems with slip dependent friction, Discrete Contin. Dyn. Syst. Ser. S 1 (2008), 117-126.

[10] S. Migórski, A. Ochal and M. Sofonea, Weak solvability of a piezoelectric contact problem, European J. Appl. Math. 20 (2009), 145-167.

[11] W. Nowacki, Some general theorems of thermo-piezoelectricity, J. Thermal Stresses 1 (1978), 171-182.

[12] M. Sofonea and EL-H. Essoufi, A piezoelectric contact problem with slip dependent coefficient of friction, Math. Model. Anal. 9 (2004), 229-242.

[13] B. Tengiz and G. Tengiz, Some dynamic problems of the theory of electroelasticity, Mem. Differential Equations Math. Phys. 10 (1997), 1-53.

[14] H.F. Tiersten, On the nonlinear equations of thermoelectroelasticity, Internat. J. Engrg. Sci. 9 (1971), 587-604.

[15] Z. Lerguet, M. Shillor and M. Sofonea, A frictional contact problem for an electroviscoelastic body, Electron. J. Differential Equations 2007 (2007), no. 170, 16 pp.

[16] G. Duvaut, Free boundary problem connected with thermoelasticity and unilateral contact, in: Free boundary problems, Vol II, Ist. Naz. Alta Mat. Francesco Severi, Rome, 1980, 217-236. 
A SIGNORINI PROBLEM WITH FRICTION IN THERMO-PIEZOELECTRICITY 411

H. Benaissa

Univ Hassan 1

Laboratory MISI

26000 Settat

Morocco

E-mail: benaissa_hicham08@yahoo.fr

EL-H. Essoufi

Univ Hassan 1

Laboratory MISI

26000 Settat

Morocco

E-mail: e.h.essoufi@gmail.com

R. Fakhar

Univ Hassan 1

Laboratory LS3M

25000 Khouribga

Morocco

E-mail: rfakhar@gmail.com

Received: 20.8.3014.

Revised: 13.7.2015. 